\title{
Introduction to Social Neuroscience: Gene, Environment, Brain, Body
}

Social neuroscience is an emerging area of neuroscience that now has its own official society (http://s4sn.org/drupal/); it defines its mission as follows:

As neuroscience matures, it has become increasingly apparent that the nervous system cannot be considered as an isolated entity, without consideration of the social environments in which humans and many animal species live. We now increasingly recognize the considerable impact on brain and body function of social structures that range from dyads, families, neighborhoods and groups to cities, civilizations, and international alliances. These factors operate on the individual through a continuous interplay of neural, neuroendocrine, metabolic and immune factors on brain and body, in which the brain is the central regulatory organ and also a malleable target of these factors. Thus, social neuroscience investigates the nervous system and its manifestations at many interacting levels-from molecules to societies - and brings together multiple disciplines and methodologies to define the emergent structures that define social species, generally, and which underlie human health and behavior, in particular. It is essential to unravel this complexity as we contemplate the future welfare of life on earth. Social neuroscience is the interdisciplinary academic field devoted to understanding how biological systems implement social processes and behavior, and how these social structures and processes impact the brain and biology. A fundamental assumption underlying social neuroscience is that all social behavior is implemented biologically. The mission of the society is to serve as an international, interdisciplinary, distributed gathering place to advance and foster scientific training, research, and applications in the field for the sake of humankind.

This outlook is reminiscent of the conference on sociophysiology organized 25 years ago by the late Patricia R. Barchas, a summary of which is found in the first article of this volume. The continued growth of neuroscience since 1986 has deepened our understanding of the brain as it responds to the social and physical environment and to new methods for evaluating it, such as enhanced brain imaging; there is also a greater appreciation of cumulative effects of stressful experiences on brain and body and their interactions with each other, as well as an updated understanding of epigenetic effects on brain activity.

Social interactions, or the lack thereof, have powerful effects upon brain and body. As summarized by Cacioppo, across many social animal species, social isolation and loneliness heighten sensitivity to social threats and motivate the renewal of social connections. The effects of perceived isolation in humans share much in common with the effects of experimental manipulations of isolation in nonhuman social species: increased sympathetic tonus and activation of the 
hypothalamic-pituitary-adrenal axis; increased inflammatory tone and altered immunity; and expression of genes regulating glucocorticoid responses. Together, these effects contribute to higher rates of morbidity and mortality in older adults.

Sleep is a sensitive index of brain and body health, and men and women differ in how social interactions affect this essential function and influence brain and body health, particularly in the elderly. As discussed by Friedman, poor sleep is associated with increased inflammatory tone, as indicated by the inflammatory protein interleukin 6 . Interestingly, in older women, good social ties compensate for poor sleep. Friedman and colleagues have extended this to demonstrate gender differences in these relationships in a national sample of middle-aged and older men and women.

Strong social ties are associated with the ability to empathize. Decety discusses empathy as a basic feature of social animal species, with the most advanced forms of empathy in humans being built on core mechanisms associated with affective communication, social attachment, and parental care. Using functional neuroimaging studies, Decety describes a brain circuit that responds to the perception of others' distress, and activation of this circuit reflects an aversive response in the observer and triggers responses to inhibit aggression or prompt motivation to help. He goes on to discuss how empathy in humans is assisted by other domain-general, high-level cognitive abilities, such as executive functioning, mentalizing, and language, which expand the range of behaviors that can be driven by empathy.

The social environment affects systemic functions that include metabolic systems, obesity, and the metabolic syndrome. Socioeconomic status is associated with gradients of obesity in developed societies, such as in the United States and many in Europe, with those at the lowest end having higher body mass, on average. ${ }^{1}$ Tamashiro reviews epidemiological evidence demonstrating the association between chronic social stress and development of obesity and symptoms leading to metabolic syndrome, effects that are evident across the lifespan. She also describes results in an animal model of a social hierarchy in which lower status is associated with greater body fat.

Other manifestations of social disruption, including adverse experiences in early life, ${ }^{2}$ are substance abuse and mental health disorders. Kreek discusses these topics, focusing on the extreme marginalization of minorities in our society, accompanied by addiction and other mental health disorders. These conditions often lead to arrest, imprisonment, and parole, all of which are characterized by lasting stigmas that haunt persons suffering from any of these problems, possibly for a lifetime.

Finally, Carlson and colleagues present new information on the Experience Corps, a program that combines social support and integration, with physical activity and meaning and purpose in life, for elderly individuals who tutor and mentor elementary school children. ${ }^{3}$ The benefits of such generativity are manifested not only in slowing age-related physical health decline but also in improved mental health and cognitive function. ${ }^{4}$ The new study shows that lower socioeconomic status in the study population is associated with a slower decline in cortisol levels over the day, which is a sign of maladaptation of the HPA axis that has been found in depression and metabolic disorders. ${ }^{5,6}$ This finding paves the way for studies assessing the ability of the Experience Corps to reverse these biological indices of unhealthy aging. This would complement and enrich the information already in hand.

This collection of articles offers the reader a flavor of current and past research at the interface between social sciences and neurobiology. But much remains to be accomplished. Can we understand the fundamental mechanisms whereby the social environment can affect brain health and disease? Can we create relevant animal models that allow us to dissect the problem at a more mechanistic level without sacrificing the richness of the social milieu and its nuanced impact on brain organization and function? Can we uncover critical periods during development and across the lifespan when the neural circuits take form, mature, and continue to evolve in an adaptive rather than a maladaptive way? How can we appreciate the importance of the community and 
social context without ignoring the uniqueness of individual members, their temperament, stress reactivity, and impact of experience on them? What is the role of these social factors in psychiatric and addictive brain disorders, for example?

These and numerous other questions await thoughtful research. What this volume says, however, is that the time has arrived for us to consider brain function and dysfunction not only in the context of the individual as a whole, but especially in the context of an individual's social setting and relationships.

BruCE S. MCEwen

The Rockefeller University, New York, New York

Huda AKIL University of Michigan, Ann Arbor, Michigan

\section{References}

1. Brunner, E.J., M.G. Marmot, K. Nanchahal, et al. 1997. Social inequality in coronary risk: central obesity and the metabolic syndrome. Evidence from the Whitehall II study. Diabetologia 40: 1341-1349.

2. Anda, R.F., A. Butchart, V.J. Felitti \& D.W. Brown. 2010. Building a framework for global surveillance of the public health implications of adverse childhood experiences. Am. J. Prev. Med. 39: 93-98.

3. Fried, L.P., M.C. Carlson, M. Freedman, et al. 2004. A social model for health promotion for an aging population: initial evidence on the Experience Corps model. J. Urban Health: Bull. N.Y. Acad. Med. 81: 64-78.

4. Carlson, M.C., K.I. Erickson, A.F. Kramer, et al. 2009. Evidence for neurocognitive plasticity in at-risk older adults: the experience corps program. J. Gerontol. A Biol. Sci. Med. Sci. 64: 1275-1282.

5. Van Cauter, E. \& F.W. Turek. 2000. Roles of sleep-wake and dark-light cycles in the control of endocrine, metabolic, cardiovascular, and cognitive function. In Coping with the Environment: Neural and Endocrine Mechanisms. pp. 313-330. Oxford University Press. New York.

6. Young, E.A., R.F. Haskett, L. Grunhaus, et al. 1994. Increased evening activation of the hypothalamic-pituitary-adrenal axis in depressed patients. Arch. Gen. Psychiatry 51: 701-707. 